EPJ Web of Conferences 100, 04004 (2015)

DOI: $10.1051 /$ epjconf/ 201510004004

(C) Owned by the authors, published by EDP Sciences, 2015

\title{
Comparison of a designed virtual oscilloscope with a real oscilloscope
}

\author{
Gozde Tektas a and Cuneyt Celiktas \\ Ege University, Faculty of Science, Physics Department, 35100, Bornova, Izmir, Turkey
}

\begin{abstract}
A virtual oscilloscope based on LabVIEW software was designed. Sinus, square and triangle shaped signals produced by a function generator were analyzed with a real and a virtual oscilloscope. Amplitude, rise time and fall time values of a signal were determined for different time/division values in both type oscilloscopes. Obtained values in the virtual oscilloscope were compared with those of the real oscilloscope. It was deduced from the results that amplitude, rise time and fall time values and signal shapes were compatible with each other.
\end{abstract}

\section{Introduction}

LabVIEW, Laboratory Virtual Instrument Engineering Workbench, is a programming environment in which programs are created by using a graphical notation. It is based on graphical programming. LabVIEW software can command plug-in data acquisition devices to acquire or generate signals. It also facilitates data transfer over a GPIB (General Purpose Interface Bus) or a serial port [1]. Its graphical nature makes it ideal for test and measurement, instrument control, data acquisition and data analysis applications [2].

An oscilloscope is a voltage sensitive electronic instrument that is used to visualize certain voltage signals. An oscilloscope can display the variation of a voltage signal in time on the oscilloscope's screen [3]. Time and amplitude values of the signal can be determined by means of the oscilloscope.

A virtual instrument consists of a computer, a software and a hardware. They are combined and configured to emulate the function of traditional hardware instrumentation. Virtual instruments are extremely flexible, powerful and cost-effective [1].

An oscilloscope can be used for amplitude, rise time and fall time measurements of a signal. The amplitude is the height of a pulse in volt unit as measured from its maximum value to its instantaneous baseline. The rise time is the time it takes for the pulse to rise from 10 to $90 \%$ of its full amplitude. The fall time is the time it takes for the pulse to fall from 90 to $10 \%$ of its full amplitude [4].

Since a virtual oscilloscope which is a kind of virtual instruments can be developed by LabVIEW software, it was designed through the program in this study. Sinus, square and triangle shaped signals were analyzed with a real and a virtual oscilloscope. Amplitude, rise time and fall time values of the signals obtained from the real and virtual oscilloscopes were compared. According to the obtained results, the virtual oscilloscope was in highly compatible with the real oscilloscope in terms of amplitude and time measurements and the signal shapes.

\section{Material and methods}

In this study, a GW Instek 2204 type oscilloscope as a real oscilloscope and a Hung Chang sweep function generator (9205C) as a signal source were used. Block diagram for the measurement is shown in Figure 1.

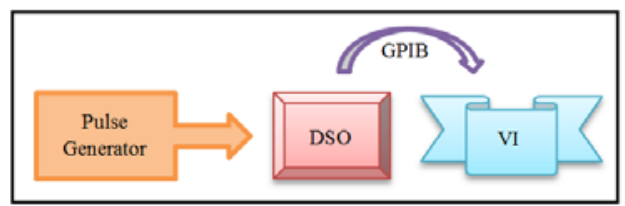

Figure 1. Block diagram for the measurement. (DSO: Digital Storage Oscilloscope, VI: Virtual Instrument, GPIB: General Purpose Interface Bus).

As can be seen in the block diagram, after sinus, triangle and square signals which were selected alternately from the function generator were displayed in the real oscilloscope; they were transferred from the real oscilloscope to the virtual oscilloscope by GPIB connection. Time/division values ranged from $1 \mu$ s to 250 $\mu$ s of the real and the virtual oscilloscopes were analyzed. During the measurement, volt/division value of each oscilloscope was kept steady on $2 \mathrm{~V}$. Frequency of the function generator was set about $155 \mathrm{kHz}$ for the $1.0,2.5$, 5.0 and $10 \mu$ s time/division values. Since the signals stayed out of the oscilloscope screen for the $25,50,100$ and 250 $\mu$ s time/division values, its frequency was decreased about to $13 \mathrm{kHz}$. In both oscilloscopes, time/division values were switched separately, and then amplitude, rise time and fall time values of the signals were determined.

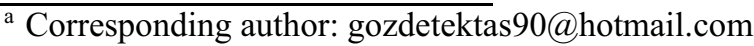


For $25 \mu \mathrm{s}$ time/division value, as an example, signal images obtained from the virtual and the real oscilloscope are shown in Fig. 2.

Sinus signal was first selected from the generator. Amplitude, rise time and fall time values of the signal versus different time/division values ranging from $1.0 \mu \mathrm{s}$ to $250 \mu$ s are given in Table 1 .
Triangle signal was secondly used. Obtained data from the real and the virtual oscilloscopes are presented in Table 2 .

Finally, the square signal was displayed in the virtual and the real oscilloscopes, and the data from the oscilloscopes can be seen in Table 3 .

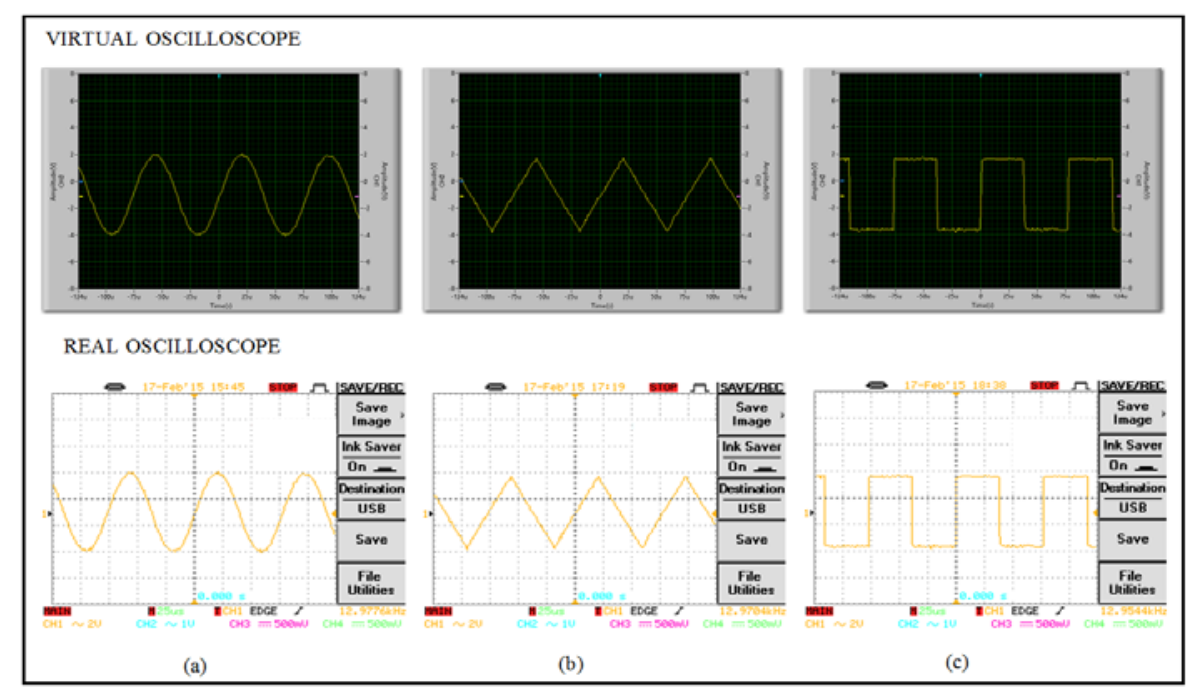

Figure 2. (a) Sinus, (b) triangle and (c) square signal shapes for $25 \mu \mathrm{s} \mathrm{time/division} \mathrm{in} \mathrm{the} \mathrm{virtual} \mathrm{oscilloscope} \mathrm{and} \mathrm{the} \mathrm{real}$ oscilloscope.

Table 1. Amplitude $\left(\mathrm{V}_{\mathrm{amp}}\right)$, rise time $\left(\mathrm{T}_{\mathrm{R}}\right)$ and fall time $\left(\mathrm{T}_{\mathrm{F}}\right)$ values for the sinus signal in the real and the virtual oscilloscopes.

\begin{tabular}{|c|c|c|c|c|c|c|}
\hline \multirow{2}{*}{ Time/Division $(\mu \mathrm{s})$} & \multicolumn{3}{|c|}{ Real Oscilloscope } & \multicolumn{3}{c|}{ Virtual Oscilloscope } \\
\cline { 2 - 7 } & $\mathrm{V}_{\text {amp }}(\mathrm{V})$ & $\mathrm{T}_{\mathrm{R}}(\mu \mathrm{s})$ & $\mathrm{T}_{\mathrm{F}}(\mu \mathrm{s})$ & $\mathrm{V}_{\text {amp }}(\mathrm{V})$ & $\mathrm{T}_{\mathrm{R}}(\mu \mathrm{s})$ & $\mathrm{T}_{\mathrm{F}}(\mu \mathrm{s})$ \\
\hline 1.0 & 5.760 & 1.824 & 1.772 & 5.760 & 1.824 & 1.772 \\
\hline 2.5 & 5.760 & 1.883 & 1.800 & 5.760 & 1.883 & 1.800 \\
\hline 5.0 & 5.760 & 1.864 & 1.799 & 5.759 & 1.864 & 1.798 \\
\hline 10.0 & 5.760 & 1.938 & 1.929 & 5.760 & 1.938 & 1.929 \\
\hline 25.0 & 5.680 & 21.750 & 20.860 & 5.681 & 21.750 & 20.870 \\
\hline 50.0 & 5.840 & 21.810 & 21.980 & 5.840 & 21.820 & 21.990 \\
\hline 100.0 & 5.760 & 21.330 & 22.300 & 5.760 & 21.340 & 22.300 \\
\hline 250.0 & 5.840 & 24.240 & 23.990 & 5.840 & 24.250 & 23.990 \\
\hline
\end{tabular}

Table 2. Amplitude $\left(\mathrm{V}_{\mathrm{amp}}\right)$, rise time $\left(\mathrm{T}_{\mathrm{R}}\right)$ and fall time $\left(\mathrm{T}_{\mathrm{F}}\right)$ values for the triangle signal in the real and the virtual oscilloscopes.

\begin{tabular}{|c|c|c|c|c|c|c|}
\hline \multirow{2}{*}{ Time/Division $(\mu \mathrm{s})$} & \multicolumn{3}{|c|}{ Real Oscilloscope } & \multicolumn{3}{c|}{ Virtual Oscilloscope } \\
\cline { 2 - 7 } & $\mathrm{V}_{\mathrm{amp}}(\mathrm{V})$ & $\mathrm{T}_{\mathrm{R}}(\mu \mathrm{s})$ & $\mathrm{T}_{\mathrm{F}}(\mu \mathrm{s})$ & $\mathrm{V}_{\text {amp }}(\mathrm{V})$ & $\mathrm{T}_{\mathrm{R}}(\mu \mathrm{s})$ & $\mathrm{T}_{\mathrm{F}}(\mu \mathrm{s})$ \\
\hline 1.0 & 4.960 & 2.388 & 2.424 & 4.960 & 2.388 & 2.424 \\
\hline 2.5 & 3.840 & 1.871 & 1.927 & 3.840 & 1.871 & 1.927 \\
\hline 5.0 & 5.120 & 2.504 & 2.431 & 5.120 & 2.504 & 2.431 \\
\hline 10.0 & 4.720 & 2.255 & 2.218 & 4.720 & 2.255 & 2.218 \\
\hline 25.0 & 3.440 & 20.260 & 19.800 & 3.440 & 20.270 & 19.800 \\
\hline 50.0 & 4.320 & 24.900 & 23.940 & 4.320 & 24.900 & 23.940 \\
\hline 100.0 & 4.080 & 22.920 & 24.070 & 4.080 & 22.920 & 24.070 \\
\hline 250.0 & 3.760 & 21.360 & 21.980 & 3.759 & 21.360 & 21.980 \\
\hline
\end{tabular}


Table 3. Amplitude $\left(\mathrm{V}_{\mathrm{amp}}\right)$, rise time $\left(\mathrm{T}_{\mathrm{R}}\right)$ and fall time $\left(\mathrm{T}_{\mathrm{F}}\right)$ values for the square signal in the real and the virtual oscilloscopes.

\begin{tabular}{|c|c|c|c|c|c|c|}
\hline \multirow{2}{*}{ Time/Division $(\mu \mathrm{s})$} & \multicolumn{3}{|c|}{ Real Oscilloscope } & \multicolumn{3}{c|}{ Virtual Oscilloscope } \\
\cline { 2 - 7 } & $\mathrm{V}_{\text {amp }}(\mathrm{V})$ & $\mathrm{T}_{\mathrm{R}}(\mu \mathrm{s})$ & $\mathrm{T}_{\mathrm{F}}(\mu \mathrm{s})$ & $\mathrm{V}_{\text {amp }}(\mathrm{V})$ & $\mathrm{T}_{\mathrm{R}}(\mu \mathrm{s})$ & $\mathrm{T}_{\mathrm{F}}(\mu \mathrm{s})$ \\
\hline 1.0 & 5.20 & 76.68 & 86.85 & 5.20 & 76.69 & 86.83 \\
\hline 2.5 & 5.28 & 143.40 & 144.20 & 5.28 & 143.40 & 144.20 \\
\hline 5.0 & 5.20 & 165.00 & 162.50 & 5.20 & 165.10 & 162.50 \\
\hline 10.0 & 5.20 & 550.90 & 550.00 & 5.20 & 550.90 & 549.80 \\
\hline 25.0 & 5.20 & 787.80 & 787.80 & 5.20 & 787.90 & 787.90 \\
\hline 50.0 & 5.28 & 1625.00 & 1600.00 & 5.28 & 1625.00 & 1600.00 \\
\hline 100.0 & 5.28 & 3200.00 & 3200.00 & 5.28 & 3200.00 & 3200.00 \\
\hline 250.0 & 5.28 & 8123.00 & 8123.00 & 5.28 & 8124.00 & 8124.00 \\
\hline
\end{tabular}

\section{Results and discussion}

Sinus, triangle and square signals from a function generator were used to compare the results from both real and virtual oscilloscopes. Amplitude, rise time and fall time values of the signals were determined from both oscilloscopes. According to the results in the Table 1, Table 2 and Table 3, amplitude, rise time and fall time values from the virtual oscilloscope were highly accorded with those of the real oscilloscope. Besides, it was observed that signal shapes in both type oscilloscopes were mostly the same as each other. It was concluded that the designed virtual oscilloscope can be used as a real oscilloscope for the determination of amplitude, rise time and fall time values.

\section{References}

1. J. Travis and J. Kring, LabVIEW for Everyone: Graphical Programming Made Easy and Fun (Third Edition, Prentice Hall, U.S.A., 2006)

2. R. Bitter, T. Mohiuddin and M. Nawrocki, LabVIEW Advanced Programming Techniques (Second Edition, Taylor and Francis Group, Boca Raton, Florida, 2007)

3. Notes on Oscilloscope, http://www.eee.metu.edu.tr/ ee214/documents/Note sOnOscilloscopes.pdf

4. R.W. Leo, Techniques for Nuclear and Particle Physics Experiments (Springer-Verlag Berlin Heidelberg, Germany, 1987) 